\title{
PHYTOSYNTHESIS OF SILVER NANOPARTICLES USING E.CAMALDULENSIS LEAF EXTRACT AND THEIR CHARACTERIZATION
}

\author{
TABASSUM BASHIR*, MUHAMMAD ZAHID QURESHI
}

Department of Chemistry, Government College University, Lahore-54000, Pakistan.

\begin{abstract}
Herein, we report a simple green synthesis of silver nanoparticles (Ag NPs) by the reduction of aqueous silver salt solution using leaf broth of Eucalyptus camaldulensis. The profile of synthesized silver nanoparticles was evaluated by using UV-visible spectrophotometer, X-ray diffractometer, atomic force microscope, energy-dispersive X-ray spectroscope and scanning electron microscope. Surface plasmon resonance peak of silver nanoparticles appeared at $425 \mathrm{~nm}$ in UV-vis spectra of silver nanoparticles. XRD studies clearly confirmed the crystalline nature of the synthesized nanoparticles. The EDX analysis disclosed the arranged inorganic composition of the synthesized Ag NPs. Atomic force microscopy investigation revealed 3D surface profile of nanoparticles. From the SEM images, the silver nanoparticles were found to be more or less spherical with an average diameter range of 110-250 nanometers.
\end{abstract}

Keywords: Ag NPs, AFM, XRD, E. camaldulensis, SEM, UV-visible spectrophotometry.

\section{INTRODUCTION}

Nanotechnology is a growing field of practical science. It deals with the materials whose structural feature express significantly unique physical, chemical, and biological properties. Nanoparticles exhibit distinctive visible properties relative to bulk material and produce quantum effects. ${ }^{1-3}$ The application of nanosilver usually ranging from 1 to $100 \mathrm{~nm}$, is uprising area of nanoscience. Silver nanoparticles have fast increased due to their chemical, optical, electronic, catalytic, magnetic and biological labeling properties. ${ }^{4-6}$ Different chemical and physical strategies are used for fabrication of silver nanoparticles, reduction in solution, thermal decomposition of silver compounds, microwave assisted laser mediated synthesis and biological reduction method. The most preferred path of silver nanoparticles synthesis offers one step eco-friendly way of synthesis. Plant-mediated synthesis of metal nanoparticle is considered a widely acceptable method for rapid production of nanoparticles..$^{7-9}$ The formation of nanoparticles was proposed to occur through the ionic or electrostatic interactions between the functional groups on the biomass surface and the metal complexes. Several bioorganic compounds in plant systems such as flavonoids, terpenoids, alkaloids, reducing sugars and proteins were suggested to be involved as either reducing or stabilizing agents during the formation of nanoparticles and their concentrations are critical in the shape directing process. ${ }^{10-12}$ The properties of silver nanoparticles totally based on their size, shape, crystallinity and structure. ${ }^{13}$ These unique characteristics of nanoparticles make them useful in the field of electronics, catalysis, biomedical analysis and even groundwater decontamination. ${ }^{14-15}$

The present study is conducted to synthesize and characterize silver nanoparticles from widely available Pakistani plant Eucalyptus camaldulensis belonging to the family, Myrtaceae. It is used for remedy of bacterial infection of the lung and urinary tracts. Volatile oils of the plants are used as expectorants while essential oil used in lung diseases. ${ }^{16}$ Topical ointments containing eucalyptus oil have also been used in ointment or cream to heal wounds and fungal infections. ${ }^{17}$ The aim of study is to develop a novel approach for the synthesis of silver nanoparticles using the aqueous leaf extract of Eucalyptus camaldulensis. This is a simple, green and cost effective method for the rapid and facile synthesis of silver nanoparticles. The work adds to authentication of previous reports on phytosynthesis of nanometals. To the best of our knowledge, this is the first report regarding synthesis of nanoparticles using aqueous leaf extract of Eucalyptus camaldulensis.

\section{MATERIALS AND METHODS}

\subsection{Preparation of plant extract}

Eucalyptus camaldulensis leaves were collected from tree at the GCU Lahore Pakistan. Leaves were first washed thrice with tap water and then washing was repeated with distilled water in order to make them dust free. The material was further dried. After drying, it was cut into small pieces and boiled with $100 \mathrm{~mL}$ distilled water in conical flask for $15 \mathrm{~min}$. Boiled mixture was filtered to separate aqueous extract which was stored at room temperature for further proceedings.

\subsection{Synthesis of silver nanoparticles}

For the synthesis of silver nanoparticles, leaf extract of E. camaldulensis was mixed with $100 \mathrm{~mL}$ of $1.0 \mathrm{mM}$ aqueous solution of silver nitrate for reduction of silver ion into silver atom. The mixture was aged for 2 hours at room temperature.

2.3. UV-Visible spectrophotometry

The reduction of pure $\mathrm{Ag}^{+}$ions was monitored by measuring the UV-Vis spectrum of the reaction medium after diluting a small aliquot of the sample by distilled water. UV-Vis spectral analysis was performed by using UV-Vis spectrophotometer (UV-1700 Shimadzu).

\subsection{XRD analysis of silver nanoparticles}

The colloidal solution of silver nanoparticles centrifuged at $13000 \mathrm{rpm}$ for $15 \mathrm{~min}$ and the obtained pellet were re-dispersed into deionized water. Thin layer of Ag NPs was obtained on slide after spread and dry suspension of silver nanoparticles. The composition and structure were investigated by XRD on PAN alytical X'Pert PRO X-ray diffractometer run at $40 \mathrm{Kv}$, thirty milli ampere current and $\mathrm{Cu} \mathrm{K}$ alpha radiation in a $\theta-2 \theta$, the diffractogram was made in the $2 \theta$ range of $20^{\circ}-80^{\circ}$.

\subsection{SEM, EDX and AFM studies}

Scanning electron microscope (SEM), energy-dispersive X-ray spectroscope (EDX) and atomic force microscope (AFM) were used to sketch construal picture of silver nanoparticles produced by leaf extract of $E$. camaldulensis. Size in nano meter range and shape of particles were revealed by scanning electron microscope formed image. The confirmation of elemental silver was analysed by EDX. Atomic force microgragh provides a threedimensional surface profile of silver nanoparticles.

\section{RESULT AND DISCUSSION}

\subsection{UV-Visible spectrophotometry}

UV-visible spectroscopic analysis was proceeded on mixture of leaf extract and silver nitrate solution. The recorded spectra showed peak at $425 \mathrm{~nm}$ which reflected the wavelength of surface plasmon resonance of silver quantum dots. The redox reaction of silver ion and biomolecules of E. camaldulensis leaf extract was observed by the UV-Vis spectroscopy ranging from 300 to $600 \mathrm{~nm}$. The maximum absorption was obtained at $425 \mathrm{~nm}$ (Fig 1). The bioreduction of $\mathrm{Ag}$ ions in solution was monitored by periodic sampling of aliquots $(0.1 \mathrm{~mL})$ of aqueous component and measuring UV-vis spectra of the solution. There was no absorption in the range of 300-600 $\mathrm{nm}$ for only leaf extract of plant while mixture of leaf extract and silver nitrate showed a distinct peak at $425 \mathrm{~nm}$. Different reports proved that peak of Ag NPs seemed to be near said region. ${ }^{10}$

3.2. XRD and EDX

X-ray diffraction pattern (XRD) was recorded for the synthesized Ag NPs (Fig 2). The results of the XRD analysis showed 20 intense values with various degree $\left(31.802^{\circ}, 37.575^{\circ}, 43.67^{\circ}, 57.025^{\circ}\right.$ and $\left.77.175^{\circ}\right)$. These results correspond to (101), (111), (200), (511) and (311) Bragg's reflection based silver nanoparticles. The well clear and strong XRD pattern showed crystalline 
nature of silver nanoparticles, as similar $2 \theta$ intense values have been reported in literature. ${ }^{18}$ In energy dispersive X-ray spectra the peak obtained at energy of $3 \mathrm{keV}$ confirmed silver, and some weak peaks of $\mathrm{Si}, \mathrm{C}$, and $\mathrm{Cl}$ were also observed (Fig 3). The emission energy at $3 \mathrm{keV}$ illustrated the reduction of silver ion.

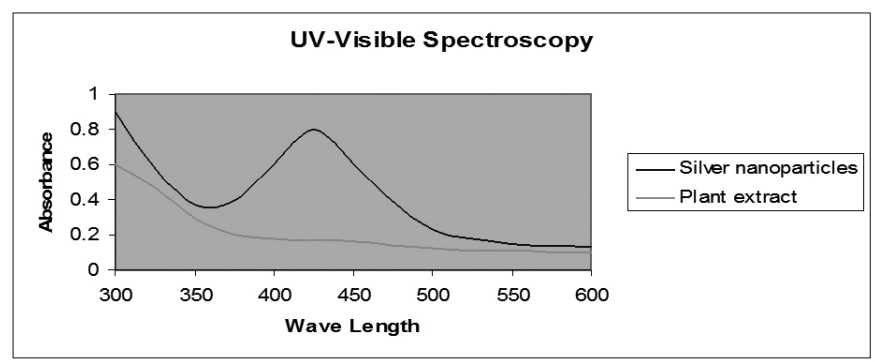

Figure $1 \mathrm{UV}$-visible spectrum of plant extract and Ag NPs synthesized by E. camaldulensis leaf extract. 3.3. Atomic Force Microscopy

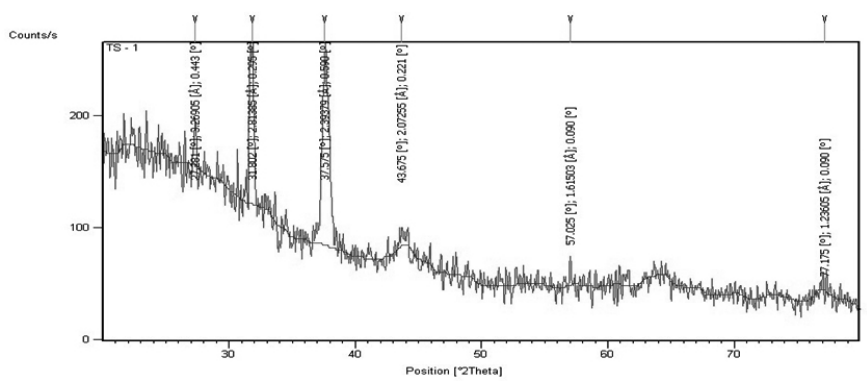

Figure 2 XRD pattern of synthesized silver nanoparticles.

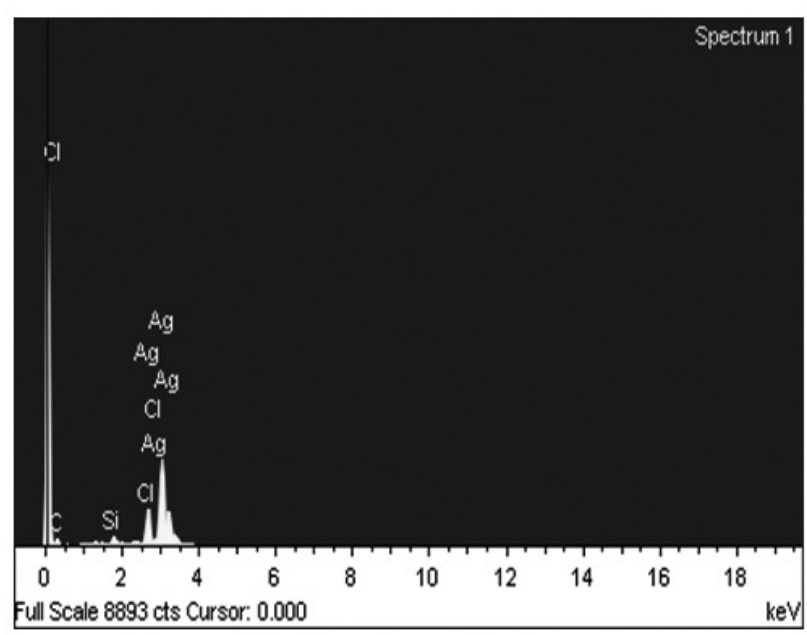

Figure 3 EDX analysis of silver nanoparticles.

Silver nanoparticles' features like surface, shape and size were studied by atomic force microscopy (Fig 4). The micrograph of the sample stated that particles were spherical and their sizes were in the range of $110-250$ nanometers.

3.4. Scanning Electron Microscopy

Scanning electron microscope (SEM) analysis of silver nanoparticles was performed using JSM-6480 SEM machine. The surface morphology of the silver nanoparticles was observed by SEM (Fig 5). The more or less spherical shape of nanoparticles was obtained with approximate size ranging $110-250 \mathrm{~nm}$. The resolution of pictures was big hurdle to measure exact sizes of nanoparticles.
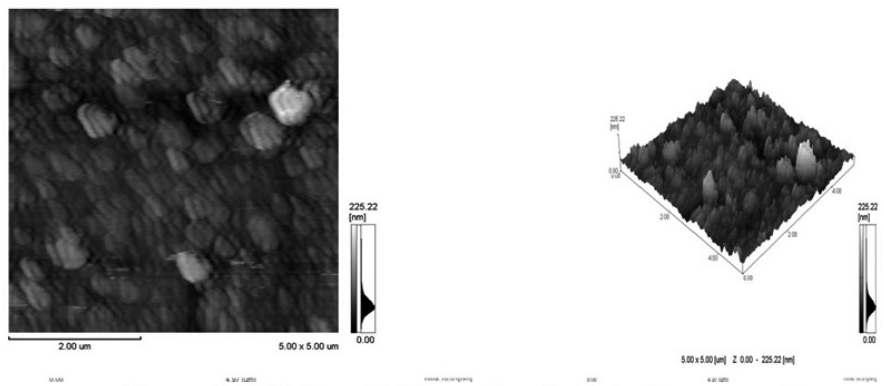

Figure 4 AFM 2D and 3D image of synthesized silver nanoparticles

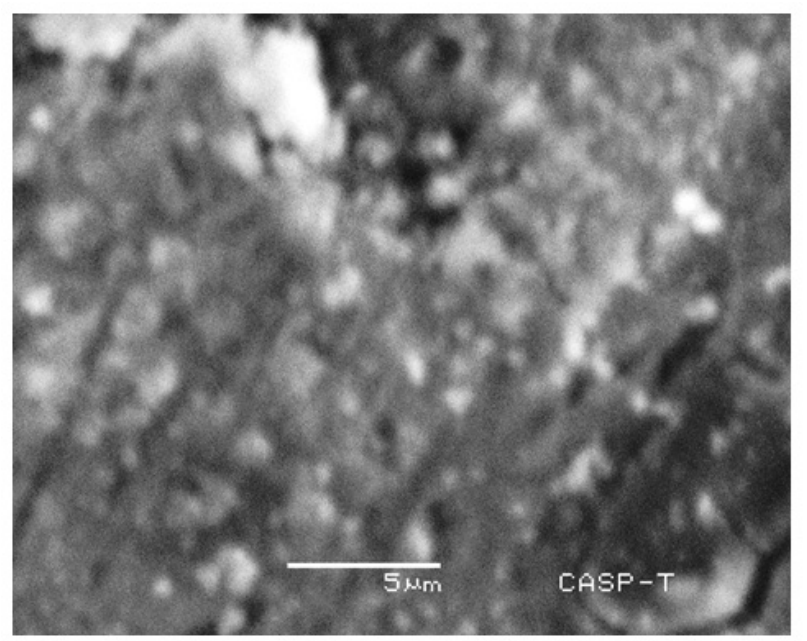

Figure 5 SEM image of silver nanoparticles using E.camaldulensis leaf extract.

\section{CONCLUSION}

E. camaldulensis extract was applied for the reduction of silver ions and green synthesis of silver nanoparticles. Silver nanoparticles were successfully synthesized with the average size ranging $110-250 \mathrm{~nm}$. The development of nanoparticles was monitored by UV-Vis spectroscopy and its results showed that most of the particles were formed during the first hour of green synthesis. This study exhibited the potential of E. camaldulensis aqueous extract for the simple and green synthesis of silver nanoparticles with several advantages such as low cost, easily available, compatibility for medical and pharmaceutical applications as well as potential for large scale production because of commercial availability of plant.

\section{REFRENCES}

1. M. Pandian, R. Marimuthu, Am. J. Nanosci. Nanotechnol. 1(2), 57, (2013)

2. M.Vanaja, G.Gnanajobitha, Journal Of Nanostructure in Chemistry. 3,17 , (2013)

3. Mujeeb Khan, M.Khan, S.F.Adil, International Journal of Nanomedicine. $8,1508,(2013)$

4. K. Gopinath, S. Gowri, J. Nanostructure Chem. 3, 68, (2013)

5. A.Kumar,C.Astalakshmi, A.Nima, International Journal of Advanced Research. 1(5), 265, (2013)

6. P.Phanjom, A.Sultana, H.Sarma, Digest Journal of Nanomaterials and Biostructures.7(3), 1117, (2012)

7. M.M.H. Khalil, E.H. Ismail, Arabian J. Chem. 7(6), 1131, (2014)

8. A.M.Awwad, Nida, M.Salem, Nanoscience and Nanotechnology. 2(4), $125,(2012)$

9. S.Gayathri S.Dr. Racheal R. Daniel, International Journal of Current Research. 4(12), 509, (2012)

10. Y. Zhou, W. Lin, J. Huang, W. Wang, Y. Gao, L. Lin, Q. Li, M. Du, Nanoscale Res. Lett. 5(8), 1351, (2010)

11. V. Kathiravan, S. Ravi, S. Ashokkumar, S. Velmurugan, Spectrochimica Acta Part A:Molecular and Biomolecular Spectroscopy. 139, 200, (2014)

12. S.M.Pourmortazavi M.Taghdiri,V.Makari, Spectrochimica Acta Part A:Molecular and Biomolecular Spectroscopy. 136, 1249, (2014) 
13. K.L. Kelly, E. Coronado, L.L. Zhao, G.C. Schatz, J. Phys. Chem. B 107(3), 668, (2003)

14. D.W. Elliott, W.X. Zhang, Environ. Sci. Technol. 35(24), 4922, (2001)

15. J.C. Wang, P. Neogi, D. Forciniti, J. Chem. Phys. 125(19), 194, (2006)
16. B.A. Adeniyi, T.O. Lawal, S.B. Olaleye, J. Bio. Sci. 6(6), 1141, (2006)

17. B.C. Akin-Osanaiye, A.S. Agbaji, M.A. Dakare, J. Med. Sci. 7(4), 694, (2007)

18. M. Sathishkumar, K. Sneha, S.W. Won, C.W. Cho, S. Kim, Y.S. Yun, Colloid Surface B 73, 332, (2009). 